\title{
Depletion of Inositol Polyphosphate 4-Phosphatase II Suppresses Callosal Axon Formation in the Developing Mice
}

\author{
Liting Ji', Nam-Ho Kim', Sung-Oh Huh ${ }^{1,2, *}$, and Hae Jin Rhee ${ }^{2, *}$
}

\begin{abstract}
The corpus callosum is a bundle of nerve fibers that connects the two cerebral hemispheres and is essential for coordinated transmission of information between them. Disruption of early stages of callosal development can cause agenesis of the corpus callosum (AgCC), including both complete and partial callosal absence, causing mild to severe cognitive impairment. Despite extensive studies, the etiology of $A g C C$ remains to be clarified due to the complicated mechanism involved in generating AgCC. The biological function of PI3K signaling including phosphatidylinositol-3,4,5-trisphosphate is well established in diverse biochemical processes including axon and dendrite morphogenesis, but the function of the closely related phosphatidylinositol-3,4,-bisphosphate (PI(3,4)P2) signaling, particularly in the nervous system, is largely unknown. Here, we provide the first report on the role of inositol polyphosphate 4-phosphatase II (INPP4B), a PI(3,4)P2 metabolizing 4-phosphatase in the regulation of callosal axon formation. Depleting INPP4B by in utero electroporation suppressed medially directed callosal axon formation. Moreover, depletion of INPP4B significantly attenuated formation of Satb2-positive pyramidal neurons and axon polarization in cortical neurons during cortical development. Taken together, these data suggest that INPP4B plays a role in the regulating callosal axon formation by controlling axon polarization and the Satb2-positive pyramidal neuron population. Dysregulation of INPP4B during cortical development may be implicated in the generation of partial AgCC.
\end{abstract}

\footnotetext{
${ }^{1}$ Department of Pharmacology, College of Medicine, ${ }^{2}$ Institute of Natural Medicine, Hallym University, Chunchon 200-702, Korea

*Correspondence: hjrhee@ hallym.ac.kr (HJR); s0huh@ hallym.ac.kr $(\mathrm{SOH})$
}

Received 10 March, 2016; revised 24 March, 2016; accepted 25 March, 2016; published online 25 April, 2016

Keywords: agenesis of the corpus callosum, axon polarization, callosal axon, cortical development, inositol polyphosphate 4-phosphatase II, phosphatidylinositol-3,4,-bisphosphate

\section{INTRODUCTION}

The corpus callosum is the largest bundle of nerve fibers that connects the two cerebral hemispheres and is essential for coordination and transmission of information between them. Several developmental processes, including correct midline patterning, formation of telencephalic hemispheres, generation of callosal neurons and their axons, and axon pathfinding, must occur in a coordinated manner for the corpus callosum to form. When any one or several of these processes is interrupted, the corpus callosum can be completely or partially absent, thin, or malformed. Agenesis of the corpus callosum (AgCC), including both complete and partial callosal absence, occurs in at least 1:4000 individuals. Affected individuals experience a diverse range of neurological disorders, from relatively mild to severe cognitive deficits (Glass et al., 2008). In addition to various genetic factors, environmental factors such as toxic insults and infection can also cause AgCC. Several reports suggest that a combination of genetic mechanisms, including single-gene Mendelian, single-gene sporadic mutations, and complex genetics may be involved in generating AgCC (Paul et al., 2007). Although many studies have been conducted to address how the formation of the corpus callosum is regulated and how malformation of this structure is generated, the etiology of AgCC still needs clarification due to the complex mechanism by which corpus callosum malformation is generated.

PI3K signaling molecules, including phosphatidylinositol3,4,5-trisphosphate $(\mathrm{PI}(3,4,5) \mathrm{P} 3)$, phosphatidylinositol-4,5-bisphosphate $(\mathrm{PI}(4,5) \mathrm{P} 2)$, and their metabolizing kinases or phosphatases plays an essential role in many diverse biological processes, including regulation of cytoskeletal rearrangement, membrane expansion, and control of gene expression. In addition, PI3K signaling is also involved in regulating axonal and dendritic morphogenesis during neurodevelopment (Cosker and Eickholt, 2007). However, the biological functions of the closely related molecule, $\mathrm{PI}(3,4) \mathrm{P} 2$, particularly those in nervous system, are largely unknown.

Here, we showed for the first time that depletion INPP4B, a $\mathrm{PI}(3,4) \mathrm{P} 2$ metabolizing phosphatase, using in utero electroporation (IUE) of Inpp4b shRNA suppresses callosal axon formation by attenuating axon polarization and the formation of Satb2-positive pyramidal neurons during cortical development, suggesting that INPP4B plays a role in regulating formation of callosal axons and is implicated in generating partial AgCC. 


\section{MATERIALS AND METHODS}

\section{Animals}

Timed pregnant mice of C57BL/6N strain were purchased from Orient Bio (South Korea). Noon on the day of the vaginal plug was defined as stage embryonic day 0.5 (E0.5). Fetuses at E13.5 were used for experiments. All animal protocols were approved by the Animal Experimentation Committee at Hallym University.

\section{DNA construction}

For construction of vectors expressing shRNA for the suppression of INPP4B expression, each shRNA oligonucleotide containing targeting sequence of the Inpp $4 b$ (5'-CCAAAGAAACAGGGTCCTCTT-3' for shlnpp4b-1, 5'-CGTTCTGTTTAAGTCGGAAT-3' for shINPP4B-2) (Dahmacon, USA) and targeting sequence of scrambled control (5'-GGACCCGAAACTACAAGTCTT-3' for shScrl) was inserted into pSuppressorNeo vector (Imgenex, USA).

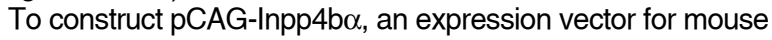
INPP4B $\alpha$ and pCAG-Inpp4b $\alpha$-GFP, full-length coding sequences of the INPP4B $\alpha$ and INPP4B $\alpha$-GFP were amplified using plnpp4bo-GFP (a kind gift from Dr. Jean Vacher, MaGill University), respectively and cloned into $\mathrm{Kpnl} /$ Notl sites of pCAG-GFP (a kind gift from Dr. C. Cepko, Harvard Medical School) after deletion of GFP fragment. For generation of shRNA-resistant forms of mouse INPP4B with silent mutation in the target sequence, the targeting site of shlnpp4b-1 was mutate into 5'-CCAAAGAGACTGGTTCCTCTT-3' from 5'-CCAAAGAAACAGGGTCCTCTT-3' by QuickChangell mutagenesis kit (Stratagene). To construct expression vector for Inpp4b$1 \mathrm{R}$, the shinpp4b-1-resistant form of INPP4B $\alpha$, the full-length coding sequences of Inpp4b-1R was amplified and clone into $\mathrm{Kpnl} /$ Notl sites of pCAG-GFP after deletion of GFP fragment. All constructs were confirmed by sequencing and their expression or shRNA efficacy was tested in transiently transfected HEK or HeLa cells before experiment.

\section{In utero electroporation}

Pregnant mice were anesthetized with isoflurane. IUE was performed essentially as described previously (Kim et al., 2014). The uterine horns were exposed by way of a laparotomy, and plasmids $(1 \mu \mathrm{l})$ containing $0.01 \%$ fast green (Sigma-Aldrich, USA) were microinjected through the uterus into the lateral ventricles of embryos by pulled glass capillaries. Electroporation was accomplished by discharging five 45V, 50-mslong pulses with $950 \mathrm{~ms}$ intervals, generated by a square-wave pulse generator (ECM 830; BTX, USA). The pulses were delivered using $5 \mathrm{~mm}$ diameter Tweezertrodes (BTX, USA). The uterine horns were then returned into the abdominal cavity, the wall and skin were sutured, and embryos were allowed to continue their normal development until the desired time of observation.

\section{Immunohistochemistry}

Electroporated embryonic brains were fixed with $4 \%$ paraformaldehyde at $4^{\circ} \mathrm{C}$ for $2 \mathrm{~h}$, followed by cryoprotected in phosphate buffered saline (PBS) containing $30 \%$ sucrose at $4^{\circ} \mathrm{C}$ overnight. Brains were then embedded in Tissue-Tek OCT (Sakura Finetek, USA). Cryosections $(10 \mu \mathrm{m})$ were collected on MAScoated glass slides (Matsunami glass, Japan) in the cryosection machine (Leica Cryosta). Section on the glass slides were treated with heat in citrate buffer $(10 \mathrm{mM}, \mathrm{pH} 6.0)$ at $95^{\circ} \mathrm{C}$ for 5 min. Samples were blocked in PBS containing $5 \%$ normal don- key serum. The sections were incubated with primary antibody against GFP (Abcam; 1:1000), Satb2 (Abcam; 1:100), Ctip2 (Abcam; 1:1200), Tuj-1 (Covance, 1:1000), Tau-1 (Millipore; 1:200), or Caspase 3 (Millipore; 1:50) overnight at $4^{\circ} \mathrm{C}$, followed by appropriate secondary antibodies conjugated with Alexa Fluor 488, 594, or 647 (Jackson ImmunoResearch Laboratories; $1: 400$ ) for $2 \mathrm{~h}$ at room temperature. After washing, the specimens were mounted onto cover slips using Vectashield (Vector Laboratories, USA). Fluorescent images were acquired with a laser scanning confocal microscope (LSM 710; Zesis) or a fluorescent microscope (Olympus IX70).

\section{Cell culture}

HEK cells were maintained in DMEM supplemented with $10 \%$ FBS (Hyclone, USA), $50 \mathrm{U} / \mathrm{ml}$ penicillin and $50 \mu \mathrm{g} / \mathrm{ml}$ streptomycin. In utero electroporation of pCAG-GFP or pCAGmCherry (Addgene) into the lateral medial cortex of E13.5 mouse embryos was performed and primary cortical neurons were prepared at E16. The cerebral cortices containing the electroporated cells were dissected manually under a dissecting fluorescence microscope. After removal of the meninges, the cortical tissue fragments were collected, digested with trypsin, and triturated in HBSS supplemented with DNasel, $10 \%$ BSA, and $10 \%$ FBS. Dissociated primary cortical neurons were initially cultured in Minimal Essential Medium (MEM) containing $10 \%$ FBS and switched to Neurobasal medium (Invitrogen) supplemented with B27 and glutamine $4 \mathrm{~h}$ after plating. The neurons were plated on coverslips coated with poly-D-lysine (Sigma) at a concentration of 40,000 cells/well in 48 well plate and cultivated at $37^{\circ} \mathrm{C}$ up to 5 days in vitro (DIV5) in humidified incubator with $5 \% \mathrm{CO}_{2}$.

\section{Immunocytochemistry}

Cells were fixed at indicated time in $4 \%$ paraformaldehyde for $10 \mathrm{~min}$ and permeabilized with $0.1 \%$ Triton $\mathrm{X}-100$ for $5 \mathrm{~min}$ at room temperature. For staining with anti-PI(3,4)P2 antibody, cells were permeabilized with $0.5 \%$ saponin (Sigma) for $15 \mathrm{~min}$. After washing with PBS, the cells were blocked with 5\% donkey serum in PBS for $1 \mathrm{~h}$ and incubated with primary antibody against GFP (Abcam, USA; 1:1000), Tau-1 (Millipore; 1:200), MAP2 (Millipore; 1:2000), INPP4B (Abcam; 1:50), and PI(3,4) $\mathrm{P} 2$ (Echelon, 1:200) overnight in $4^{\circ} \mathrm{C}$. Appropriate secondary antibodies were incubated for $45 \mathrm{~min}$ at room temperature, and specimens were prepared as described in Immunohistochemistry. To label polymerized actin (F-actin), phalloidin-conjugated Alexa Fluor 647 (Invitrogen) were incubated together with secondary antibodies.

\section{Western blot analysis}

Western blot was performed essentially as described previously (Kim et al., 2014). HEK cells were lysed in lysis buffer (50 mM Tris- $\mathrm{HCl}, \mathrm{pH}$ 7.5, $150 \mathrm{mM} \mathrm{NaCl}, 1$ mM EGTA, 1 mM EDTA, $1 \%$ Triton X-100, $1 \mathrm{mM} \mathrm{Na}_{3} \mathrm{VO}_{4}, 5 \mathrm{mM} \mathrm{NaF}$, and protease inhibitor cocktail). After incubation on ice for $30 \mathrm{~min}$, the lysates were centrifuged. Supernatants were collected and protein concentrations were determined by Bradford assay (Bio-Rad, USA). Equal amounts of protein were loaded and separated by SDS-PAGE (8\% reducing gels), transferred to polyvinylidene difluoride membranes (Millipore, USA), and blocked with $5 \%$ non-fat milk. Membranes were incubated in primary antibody against GFP overnight at $4^{\circ} \mathrm{C}$. Membranes were then washed in TBST (10 mM Tris, $140 \mathrm{mM} \mathrm{NaCl}, 0.1 \%$ Tween 20, pH 7.6), incubated with appropriate secondary antibody, and washed again in TBST. Bands were visualized by enhanced chemilu- 
A

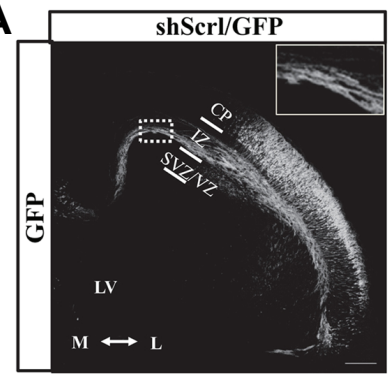

B

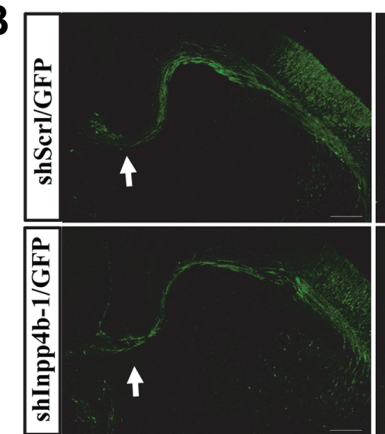

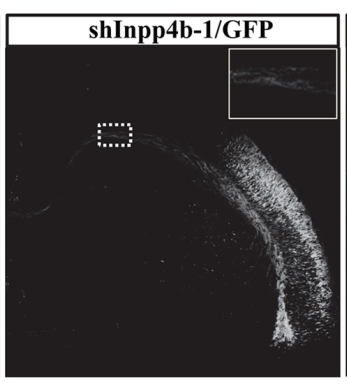
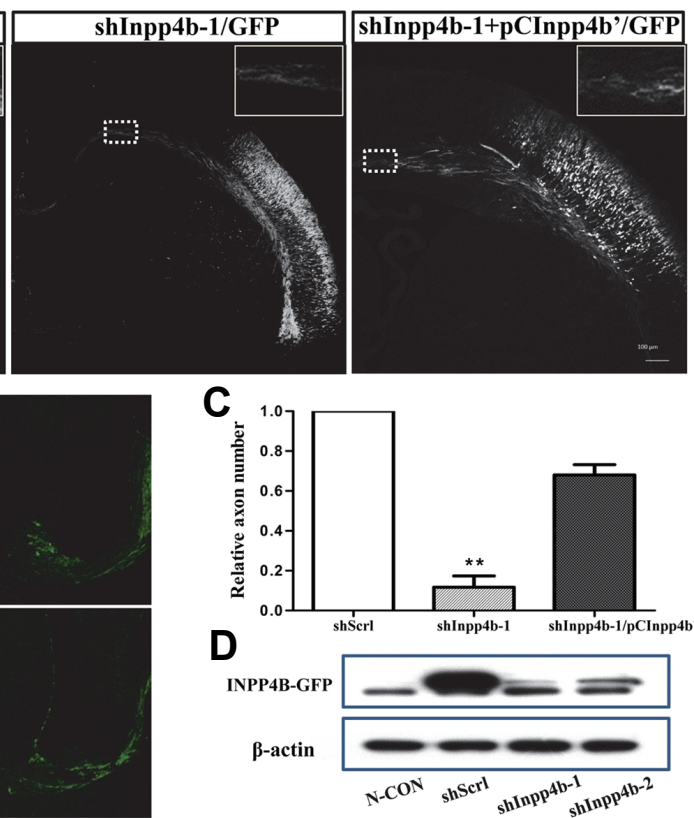

C

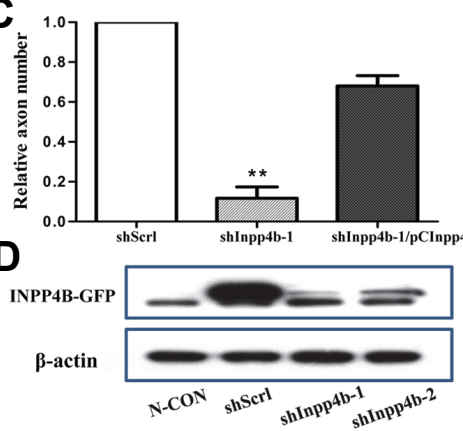

Fig. 1. Depleting INPP4B reduces callosal axon formation. (A) shlnpp4b-1 or shScrl together with pCAG-GFP was injected into the lateral ventricles of mouse embryos for in utero electroporation at E13.5 to knockdown Inpp4b. For the rescue experiment, pCAG-Inpp4b-1R (pCInpp4b') (1 $\mu \mathrm{g})$, a vector expressing the shlnpp4b-1-resistant form of INPP4B $\alpha$ and shlnpp4b-1 $(1 \mu \mathrm{g})$ together with pCAG-GFP $(0.4 \mu \mathrm{g})$ were injected. Mouse embryos were collected at E17.5 and coronal brain sections were prepared. GFP fluorescent images of each sample were acquired with a confocal microscope $(10 \times$, tile scan) under the same detection condition. Enlarged fluorescent image in the regions marked with the white dotted line was shown at the topright corner. Scale bars represent $200 \mu \mathrm{m}$ (B) Maximum intensity projections of confocal z-stacks of coronal sections of mouse embryos electroporated with shlnpp4b-1 or shScrl along with pCAG-GFP at E13.5. Cryosections $(10 \mu \mathrm{m})$ were prepared at

E17.5 and stained with anti-GFP antibody. Fluorescent images of the coronal sections were acquired with a confocal microscope (20x, zoom 0.6x). Callosal axons of electroporated neurons reached the midline (arrow). Enlarged fluorescent image around the midline is shown in right panel. (C) The relative number of axon bundles in the callosal projections inside the dotted box [as shown in (A)] was quantified and summarized in the bar graph. Data are mean \pm SEM from section images of three separate embryo pairs. Each embryo pairs was derived from same mouse. Data were evaluated with the $t$-test $\left(n=3,{ }^{* *} p<0.001\right.$ vs control). (D) Evaluation of shlnpp4b the knockdown efficiency. Lysates from HEK cells transfected with shScrl, shlnpp4b-1, or shlnpp4b-2, together with pCAG-Inpp4bo-GFP were prepared and applied to a Western blot analysis. Mouse INPP4B fused to GFP was detected using anti-GFP antibody. Lysates from HEK cells transfected with an empty vector alone were used as a negative control (N-CON).

minescence and exposed to X-ray film.

\section{Determination of knockdown efficiency}

The knockdown efficiency of shlnpp4b was determined by Western blot analysis of HEK cells transfected with pCAGInpp4bo-GFP along with shINPP4B or shScrl, a control knockdown vector. HEK cells were transfected by using Fugene HD (Roche).

\section{Image analysis and statistical methods}

Neuronal morphological transition after in utero electroporation was determined using at least three different embryos and at least 36 sections. After staining section with 4',6-diamidino2phenylindole (DAPI) and anti-Tuj-1, the cerebral cortex was subdivided into ventricular zone (VZ), subventricular zone (SVZ), intermediate zone (IZ), and cortical plate (CP) based on cell density and Tuj-1 signal intensity.

Relative number of axon bundles was determined by measuring fluorescent intensity of transfected GFP in the callosal projections axons in selected regions, which was normalized to total GFP intensity of transfected cells in the cerebral cortex. For quantitative comparison of GFP signal, total fluorescent intensity of GFP in each sample set was acquired under the same detection condition with the Zeiss LSM 710 confocal microscopy.

Measuring axon length and neurite branch of the cortical neurons was analyzed with Neuron $\mathrm{J}$ and Neurophology $\mathrm{J}$, respectively. A cell was considered to have an axon if the length of one process was more than twice the diameter of the cell body or if this process was Tau-1-positive \& MAP2negative (Lee et al., 2014).

Results were expressed as mean \pm SEM. Statistical significances were assessed with $\chi^{2}$ test, two-tailed $t$-test, one-way analysis of variance (ANOVA), or two-way ANOVA using Prism 5 (GradPad Software, USA).

\section{RESULTS}

Depleting INPP4B suppresses callosal axon formation $\mathrm{PI}(3,4) \mathrm{P} 2$ metabolizing enzymes such as INPP4B and $\mathrm{SH} 2$ domain-containing 5-phosphatase, are expressed in the developing mouse cortex (Muraille et al., 2001), however, the biological functions of $\mathrm{PI}(3,4) \mathrm{P} 2$ in the nervous system except neuronal survival remains largely unknown. We examined the effect of Inpp4b knockdown on corticogenesis to explore the biological functions of INPP4B during cortical development. Depleting INPP4B by electroporation of shlnpp4b-1, an Inpp4b knockdown vector together with a pCAG-GFP vector to mark the electroporated cells, reduced the number of medially directed callosal projection axon (Fig. 1A). However, depleting INPP4B might not affect callosal axon outgrowth since majority of the callosal axon also outgrown normally and reached the midline in shlnpp4b-1 sample in the same way that in control (Fig. 1B). The knockdown efficiency of shlnpp4b was confirmed by Western blot in HEK cells expressing mouse INPP4B $\alpha$-GFP (Fig. 1D). To ensure specificity of the phenotype for Inpp4b knockdown, another Inpp4b shRNA, shlnpp4b-2, was electroporated and yielded a similar phenotype (data not shown). A 
A

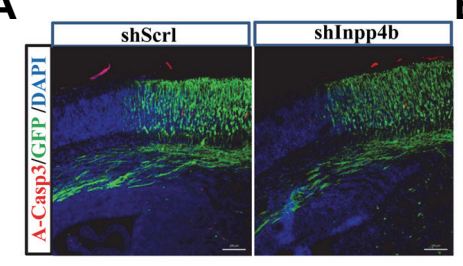

$B$

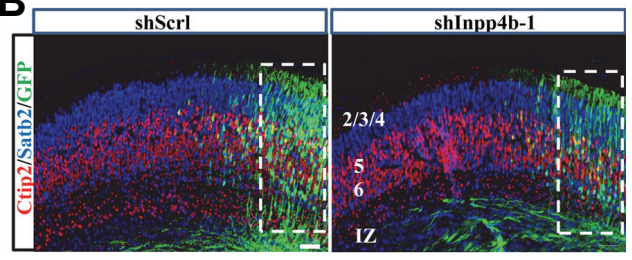

C

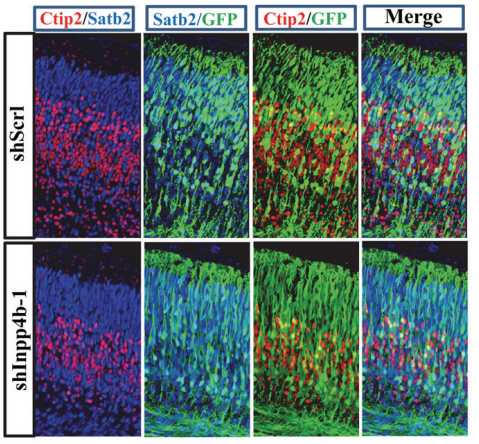

D

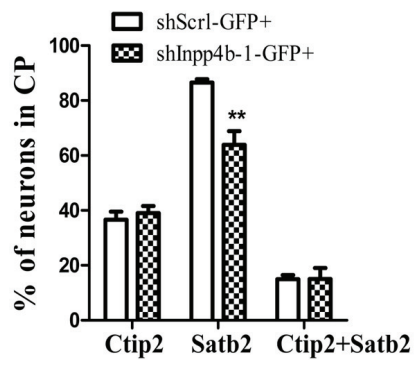

Fig. 2. INPP4B depletion diminishes the formation of SatB2-positive pyramidal neurons. Mouse embryos were electroporated in utero with shlnpp4b-1 or shScrl together with pCAG-GFP at E13.5, and collected at E17.5 to prepare coronal sections. (A) Fluorescent images of coronal sections immunostained with antibodies against GFP and activated caspase 3 (A-casp3) are representative of two independent experiments. (B) Maximum intensity projections of confocal z-stacks of coronal sections stained with antibody against GFP, Satb2, and Ctip2. The fluorescent images were acquired with a confocal microscope $(20 \times$, tile). (C) Magnified images of the selected region marked with the boxes in $B$ were shown as double- and triple-merged images, respectively. (D) Fraction of GFPpositive cortical neurons within CP stained with Ctip2, Satb2, or double positive with Ctip2 and Satb2 in Fig. 2C were quantified,

respectively and summarized in a bar graph. Each fraction was analyzed using a two-way ANOVA followed by a Bonferroni post-hoc test $\left({ }^{* \star} p\right.$ $<0.001$, vs shScrl; $n=3$ ). Scale bars, $50 \mu \mathrm{m}$.

rescue IUE experiment by co-electroporation of pCInpp4b', an expression vector for the shlnpp4b-1-resistant form of Inpp4b together with shlnpp4b-1, restored callosal axon formation nearly to normal, confirming that the phenotype is an Inpp4bspecific effect, and not the result of off-target effects (Fig. 1C).

\section{Depleting INPP4B diminishes the SatB2-positive pyramidal neuron population}

As $\mathrm{PI}(3,4) \mathrm{P} 2$ metabolism is involved in neuronal survival signaling (Nystuen et al., 2001; Sasaki et al., 2010), depletion of INPP4B may affect survival of cortical neurons responsible for callosal axon formation. We determined whether depleting INPP4B in cortical neurons affects cell survival. However, no observable difference in cell survival was detected between cortical neurons electroporated with shlnpp4B and cortical neurons electroporated with shScrl (Fig. 2A). Next we test the possibility that Inpp4b knockdown affects formation of callosal projection neurons (CPN) during cortical development, which is responsible for callosal axon formation. Satb2 is a DNA binding protein expressed abundantly in CPN and is a major regulatory determinant for CPN in the developing cerebral cortex (Alcamo et al., 2008; Britanova et al., 2008), while Ctip2 is a key determinant for extending subcortical projection by cortical neurons. CPN formation was monitored by determining Satb2 expression in the developing cortex after co-electroporation of pCAGGFP and shlnpp4b-1 or shScrl. Depleting INPP4B reduced the fraction of Satb2-positive cortical neurons in cortical plate (CP), but had little effect on that of Ctip2-positive pyramidal neurons, when INPP4B-depleted cortical neurons (shlnpp4b-1/GFP+) were compared to control (shScrl/GFP+) (Fig. 2) or cortical neurons untransfected in the same coronal section of embryos transfected with shlnpp4b (data not shown).

\section{Depleting INPP4B attenuates axon polarization in developing cortical neuron}

Several authors have shown that PI3K and PI3K-dependent regulation of ILK, Akt, and GSK-3 are involved in the regulation of axon specification and elongation (Cosker and Eickholt, 2007; Guo et al., 2007; Oinuma et al., 2007). As INPP4B is shown to be implicated in regulating callosal axon formation which can be controlled by cortical axon specification, we addressed whether INPP4B played a role in regulating cortical axon specification. The effect of depleting INPP4B on axon polarization was monitored in primary cortical neurons depleted of INPP4B ex vivo using IUE. Co-electroporation of pCAG-GFP and either shlnpp4b-1 or shScrl into the lateral ventricles of the mouse embryo generated cortical neurons depleted of INPP4B or normal neurons in vivo. Axon polarization was determined in the primary GFP-positive cortical neurons dissociated at E16 from the mouse brains co-electroporated with pCAG-GFP and either shlnpp4b-1 or shScrl at E13.5. Depleting INPP4B significantly suppressed the generation of GFP-positive neurons with axons and concomitantly increased GFP-positive neurons without axon, but did not affect axon length, branch, or neurite number (Fig. 3A).

To examine subcellular localization of INPP4B in cortical neurons, primary cortical neurons were immunostained with anti-INPP4B antibody (Abcam, USA), but did not produce a fine fluorescence image. Alternatively, INPP4B $\alpha$ localization was examined in primary cortical neurons expressing INPP4B $\alpha$ GFP. INPP4B $\alpha$-GFP was localized preferentially in dendrites compared to axons in polarized cortical neurons (Fig. 3D), whereas INPP4B $\alpha$-GFP was distributed evenly in all neurites in unpolarized cortical neurons at stage 2 or early stage 2 (data not shown). Preferential dendritic localization of INPP4B $\alpha$ in developing cortical neurons was also shown in vivo by confocal imaging of a coronal section of embryonic brain after electroporation with pCAG-Inpp4bo-GFP (Fig. 3C). To determine the local distribution of $\mathrm{F}$-actin and $\mathrm{PI}(3,4) \mathrm{P} 2$, a specific substrate of INPP4B in cortical neurons, primary cortical neurons were immunostained with phalloidin and anti-PI(3,4)P2 antibody, respectively and a quantitative profile of $\mathrm{PI}(3,4) \mathrm{P} 2$, m-Cherry, and F-actin, or INPP4B $\alpha$-GFP levels in a region of interest (ROI) in axon and dendrites in the polarized neurons were analyzed 
A
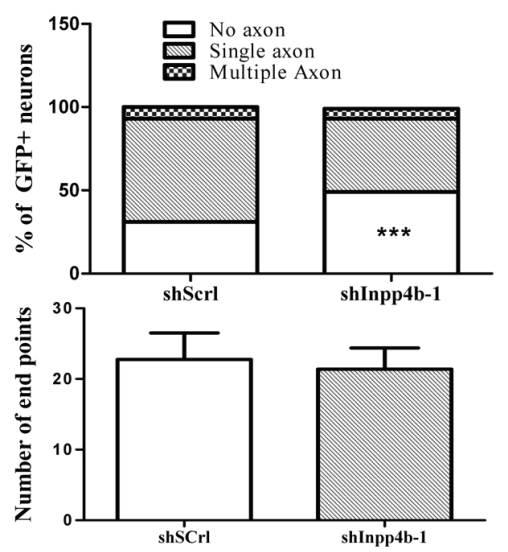

C

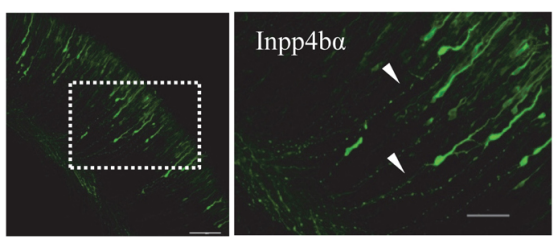

F
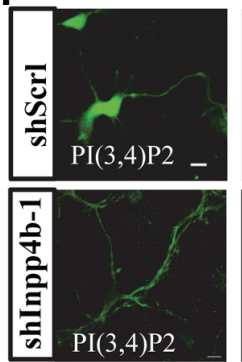
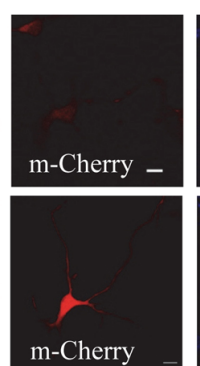

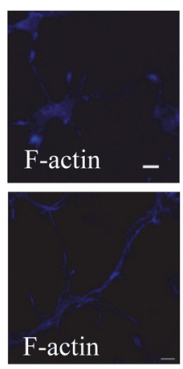

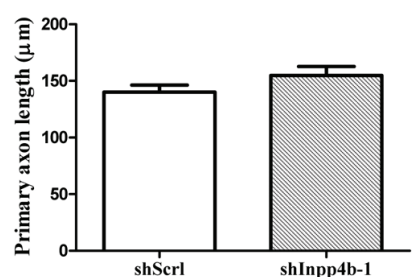

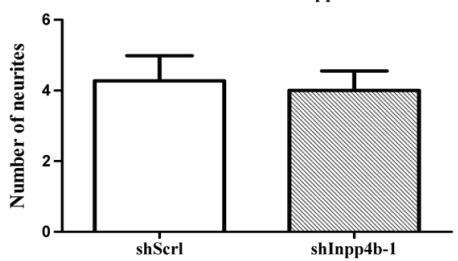

D

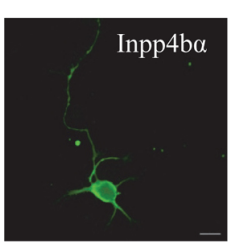

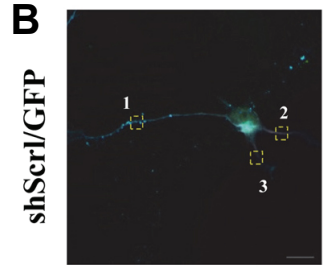
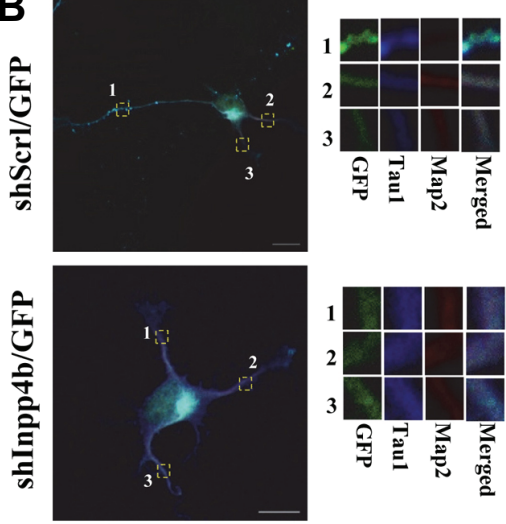

E
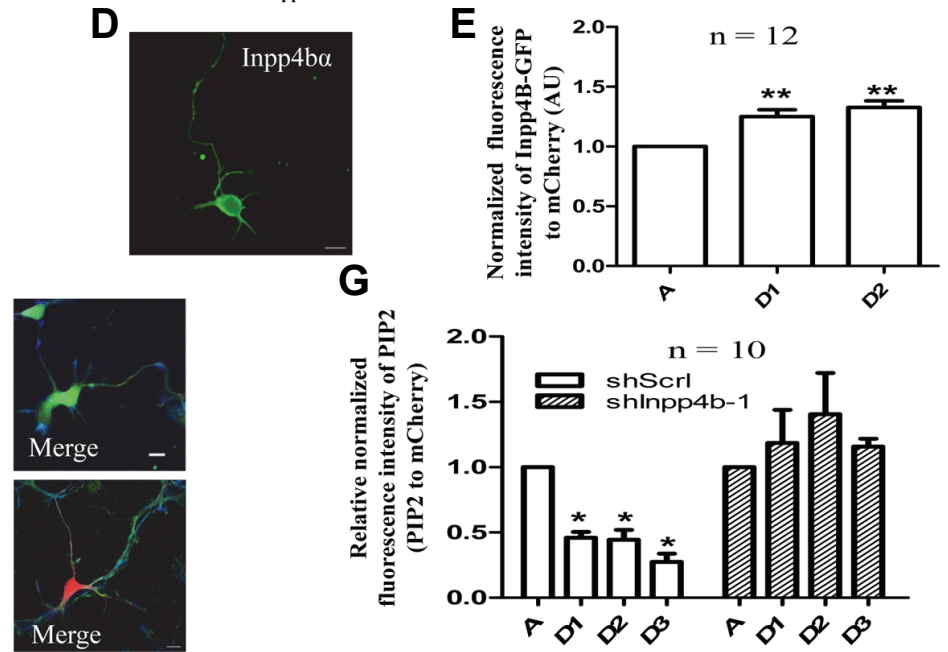

G

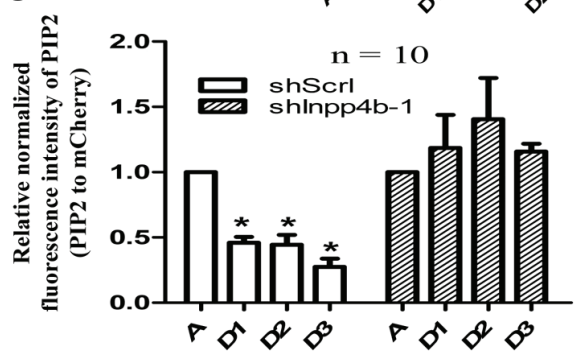

Fig. 3. INPP4B depletion suppresses axon polarization. Mouse embryos were electroporated in utero with shlnpp4b-1 or shScrl, together with pCAG-GFP or pCAG-mCherry into the lateral medial cortex at E13.5 and collected at E16. Cortical neurons electroporated with pCAG-GFP or pCAG-mCherry were cultivated for 1-3 days. (A) The cortical neurons co-electroporated with pCAG-GFP and shScrl or shlnpp4b-1were fixed at DIV2 and immunostained with antibodies against GFP, Tau-1, and MAP2. Fluorescence images were acquired with a fluorescence microscope or a confocal microscope. The fraction of GFP-positive neurons with no axon, single axon, and multiple axons were quantified in both shScrl and shlnpp4b samples in $(A)\left({ }^{* \star *} p<0.0001\right.$ vs shScrl, $X^{2}$ test; $n=221$ neurons transfected with shScrl and $n=179$ neurons transfected with shlnpp4b from at least three independent experiments). Axon length, end point number, and neurite number were determined at DIV3 ( $t$ test; $n=158$ neurons transfected with shScrl or shINPP4B). The primary axon length was measured by ImageJ. The number of end points and the number of neurites were determined by Neurophology J. (B) Representative confocal merged images of cortical neuron electroporated in utero with shlnpp4b-1 or shScrl are shown. Fluorescence image of Tau-1, MAP2, and GFP at three ROls marked with circle in each neurites were acquired and used for determination of axon as described in "Material and Methods". (C) Maximum intensity projections of confocal z-stacks of coronal sections of mouse embryos electroporated with pCAG-Inpp4bo-GFP at E13.5. Cryosections $(30 \mu \mathrm{m})$ were prepared at E17.5 and fluorescent image of the coronal sections were acquired with a confocal microscope (20x, zoom 0.6x). Preferential localization of INPP4B in the leading dendrites and cell bodies is shown compared to that in the axons (inset, white arrowheads) of pyramidal neurons. (D) Fluorescent images of primary cortical neurons electroporated pCAG-Inpp4b $\alpha$-GFP $(0.4 \mu \mathrm{g})$ along with pCAG-mCherry $(1.6 \mu \mathrm{g})$. Cells were fixed at DIV2 (E) A quantitative profile of INPP4B $\alpha$-GFP levels in a ROls in the polarized neurons shown in D were analyzed using ZEN software and relative normalized fluorescence intensity of Inpp4B-GFP to mCherry at the ROI at the axon and at the dendrites of the cortical neurons was shown $\left({ }^{* *} p<0.001\right.$ vs axon, one-way ANOVA followed by a Bonferroni test). (F) Fluorescent images of primary cortical neurons coelectroporated with pCAG-mCherry and shlnpp4b-1, or shScrl. The cells were fixed at DIV2 and immunostained with an anti-PI(3,4)P2 antibody. (G) Relative normalized fluorescence intensity of $\mathrm{PI}(3,4) \mathrm{P} 2$ to mCherry at the ROI at the axon and at the dendrites of the cortical neurons shown in $F(\# p<0.0001$, vs axon, one-way ANOVA followed by a Bonferroni test). Scale bars, (A-C), (E), ( $F), 10 \mu \mathrm{m} ;(\mathrm{D}), 100 \mu \mathrm{m}$.

using ZEN software. Consistent with dendritic localization of INPP4B (Figs. 3C-3E), PI(3,4)P2 was enriched preferentially in axons in polarized cortical neurons and preferential axonal enrichment was abolished by depleting INPP4B (Figs. 3E and 3G).

\section{DISCUSSION}

As biological functions of $\mathrm{PI}(3,4) \mathrm{P} 2$ in the nervous system remained largely unknown, we explored the effect of Inpp4b knockdown on corticogenesis in developing mouse brain. In 
this report, we showed that INPP4B plays a role in the regulation of callosal axon formation by controlling the Satb2positive pyramidal neuron population and axon polarization. Depleting INPP4B in developing cortex by IUE of shInpp4b suppressed callosal axon formation and a rescue IUE experiment using pCInpp4b', a vector expressing INPP4B $\alpha$ resistant to shlnpp4b-1 or 2, confirmed that the knockdown effect was specific for INPP4B despite the partial restoration of the CPN axon (Fig. 1). INPP4B $\alpha$ and $\beta$ are two isoforms produced by alternative splicing of the 3' end of Inpp4b in the developing mouse brain (Ferron and Vacher, 2006). As a vector expressing INPP4B $\alpha$ was only used for the rescue experiment, although shlnpp4b-1 or shlnpp4b-2 downregulated the two INPP4B isoforms due to identical shlnpp4b target sites in both Inpp4bo and Inpp4b $\beta$, this rescue result raised the possibility that INPP4B $\beta$ might be partly implicated in the regulation of callosal axon formation.

Although INPP4B, an enzyme metabolizing PI(3,4)P2 controls cell survival or proliferation, depleting INPP4B did not affect neuronal cell survival in developing mouse cortex, but suppressed formation of Satb2-positive CPN population in developing CP. Thus, this data are consistent with the observation that depleting INPP4B suppressed preferentially callosal axon formation, but marginally affects subcortical projection formation (data not shown)

Depleting INPP4B in the cortical progenitors significantly suppressed the fraction of GFP-positive neurons with axons in the primary cortical neuron culture, but did not affect axon length, branch, or neurite number (Fig. 3A). This data suggests that INPP4B may be involved in axon polarization and supports previous result showing that depleting INPP4B may not affect axon length in vivo (Fig. 1B). To address role of INPP4B in axon polarization, subcellular localization of INPP4B, PI $(3,4) \mathrm{P} 2$, and $\mathrm{F}$-actin was examined. Preferential dendritic localization of INPP4B $\alpha$ in developing cortical neurons may resulted in enriched localization of $\mathrm{PI}(3,4) \mathrm{P} 2$ in axons (Figs. 3C-3G). The polarized distribution of $\mathrm{PI}(3,4) \mathrm{P} 2$ did not show any particular correlation with F-actin localization except some specific regions enriched with F-actin, including neurite processes, axon tips, and axon collateral branches (data not shown), suggesting that an increase in $\mathrm{PI}(3,4) \mathrm{P} 2$ in these regions is involved in $\mathrm{F}$ actin-mediated morphogenesis of axon in developing cortical neurons. These data are consistent with previous observations that lamellipodin is located in the $\mathrm{PI}(3,4)$-rich region on the plasma membrane and recruits Ena/VASP proteins promoting actin polymerization and bundling during cell migration and polarization (Krause et al., 2004; Michael et al., 2010). Taken together, our data showing preferential localization of INPP4B $\alpha$ in dendrites and the subsequent polarized distribution of $\mathrm{PI}(3,4) \mathrm{P} 2$ in the axons of polarized cortical neurons during cortical development suggest that the selective increase in $\mathrm{PI}(3,4) \mathrm{P} 2$ by INPP4B or reducing localization of INPP4Binteracting proteins in axons may be involved in regulating axon specification in developing cortical neurons. Considering that the majority of $\mathrm{PI}(3,4) \mathrm{P} 2$ signals in axons were not co-localized with F-actin, the polarized axonal distribution of $\mathrm{PI}(3,4) \mathrm{P} 2$ may not be coupled with F-actin dynamics. Thus, the spatiotemporal change in localization of INPP4B $\alpha$ during axon polarization may play a role in modulating callosal axon polarization during cortical development, although the detailed mechanism is obscure. Further studies are needed to clarify the requirement for INPP4B $\alpha \mathrm{PI}(3,4) \mathrm{P} 2$ phosphatase activity in the regulation of axon formation and to determine spatiotemporal localization of activated INPP4B during axon polarization to delineate the regulating mechanism. These data shed light on a new function of INPP4B in the regulation of callosal axon formation.

In conclusion, our results showed that depleting INPP4B by IUE suppresses medially directed callosal axon formation. Moreover, depleting INPP4B significantly attenuated the generation of Satb2-positive pyramidal neurons and axon polarization in cortical neurons during cortical development. Although further studies are necessary to delineate the mechanism by which INPP4B regulates the formation of callosal axons, our data provides the first experimental evidence that INPP4B may play a role in regulating callosal axon formation by the formation of Satb2-positive pyramidal neurons and controlling axon polarization, suggesting that dysregulation of INPP4B may be implicated in the generation of partial AgCC.

\section{ACKNOWLEDGMENTS}

This work was supported by National Research Foundation of Korea Grant funded by the Korean Government (NRF-20100005848) and (NRF-2015R1A2A2A01007473), by Priority Research Centers Program through the National Research Foundation of Korea (NRF) funded by the Ministry of Education, Science and Technology (NRF-2009-0094071), and by Hallym University Research Fund (HRF-G-2015-3).

\section{REFERENCES}

Alcamo, E.A., Chirivella, L., Dautzenberg, M., Dobreva, G., Farinas, I., Grosschedl, R., and McConnell, S.K. (2008). Satb2 regulates callosal projection neuron identity in the developing cerebral cortex. Neuron 57, 364-377.

Britanova, O., de Juan Romero, C., Cheung, A., Kwan, K.Y., Schwark, M., Gyorgy, A., Vogel, T., Akopov, S., Mitkovski, M., Agoston, D., et al. (2008). Satb2 is a postmitotic determinant for upper-layer neuron specification in the neocortex. Neuron 57, 378-392.

Cosker, K.E., and Eickholt, B.J. (2007). Phosphoinositide 3-kinase signalling events controlling axonal morphogenesis. Biochem. Soc. Trans. 35, 207-210.

Ferron, M., and Vacher, J. (2006). Characterization of the murine Inpp4b gene and identification of a novel isoform. Gene 376, 152-161.

Glass, H.C., Shaw, G.M., Ma, C., and Sherr, E.H. (2008). Agenesis of the corpus callosum in California 1983-2003: a populationbased study. Am. J. Med. Genet. A. 146A, 2495-2500.

Guo, W., Jiang, H., Gray, V., Dedhar, S., and Rao, Y. (2007). Role of the integrin-linked kinase (ILK) in determining neuronal polarity. Dev. Biol. 306, 457-468.

Kim, N.H., Kim, S., Hong, J.S., Jeon, S.H., and Huh, S.O. (2014). Application of in utero electroporation of G-protein coupled receptor (GPCR) genes, for subcellular localization of hardly identifiable GPCR in mouse cerebral cortex. Mol. Cells 37, 554561.

Krause, M., Leslie, J.D., Stewart, M., Lafuente, E.M., Valderrama, F., Jagannathan, R., Strasser, G.A., Rubinson, D.A., Liu, H., Way, M., et al. (2004). Lamellipodin, an Ena/VASP ligand, is implicated in the regulation of lamellipodial dynamics. Dev. Cell. 7, 571-583.

Lee, N., Park, J.W., Kim, H.J., Yeon, J.H., Kwon, J., Ko, J.J., Oh, S.H., Kim, H.S., Kim, A., Han, B.S., et al. (2014). Monitoring the differentiation and miggration patterns of neural cells derived from human embryonic stem cells using a microfluidic culture systerm. Mol. Cells 37, 497-502.

Michael, M., Vehlow, A., Navarro, C., and Krause, M. (2010). c-Abl, Lamellipodin, and Ena/VASP proteins cooperate in dorsal ruffling of fibroblasts and axonal morphogenesis. Curr. Biol. 20, 783-791.

Muraille, E., Dassesse, D., Vanderwinden, J.M., Cremer, H., Rogister, B., Erneux, C., and Schiffmann, S.N. (2001). The SH2 domain-containing 5-phosphatase SHIP2 is expressed in the germinal layers of embryo and adult mouse brain: increased expression in N-CAM-deficient mice. Neuroscience 105, 10191030.

Nystuen, A., Legare, M.E., Shultz, L.D., and Frankel, W.N. (2001). 
A null mutation in inositol polyphosphate 4-phosphatase type I causes selective neuronal loss in weeble mutant mice. Neuron 32, 203-212.

Oinuma, I., Katoh, H., and Negishi, M. (2007). R-Ras controls axon specification upstream of glycogen synthase kinase-3beta through integrin-linked kinase. J. Biol. Chem. 282, 303-318.

Paul, L.K., Brown, W.S., Adolphs, R., Tyszka, J.M., Richards, L.J.,
Mukherjee, P., and Sherr, E.H. (2007). Agenesis of the corpus callosum: genetic, developmental and functional aspects of connectivity. Nat. Rev. Neurosci. 8, 287-299.

Sasaki, J., Kofuji, S., Itoh, R., Momiyama, T., Takayama, K., Murakami, H., Chida, S., Tsuya, Y., Takasuga, S., Eguchi, S., et al. (2010). The Ptdlns $(3,4) P(2)$ phosphatase INPP4A is a suppressor of excitotoxic neuronal death. Nature 465, 497-501. 